\title{
The proportion of lung cancer patients attending UK lung cancer clinics who would have been eligible for low-dose CT screening
}

\author{
To the Editor:
}

The National Lung Screening Trial (NLST) demonstrated that low-dose computed tomography (LDCT) screening reduced lung cancer mortality by $20 \%$ [1]. Lung cancer screening was subsequently recommended by the US Preventive Services Taskforce (USPSTF) for patients at high risk of lung cancer [2], but European nations have awaited the results of the Dutch-Belgian NELSON trial [3] before committing to national programmes. The initial findings from NELSON, presented recently at the World Conference on Lung Cancer [4], appear to confirm the reduction in lung cancer mortality, and will increase the impetus for implementation in Europe. A European position statement on lung cancer screening identified specific actions required prior to systematic LDCT screening [5]. These include the development of a risk stratification approach to identify the population for lung cancer screening, and recognition that harm-to-benefit considerations make it unethical to screen low risk patients.

The NLST identified participants for screening based on age and smoking history (55-74 years, at least 30 pack-years, and a quit time of less than 15 years for ex-smokers) [1]. The USPSTF recommended LDCT screening based on the NLST criteria and modelling studies that examined ages to begin and end screening, screening intervals and the relative benefits and harms of different screening strategies, extending the upper age limit to 80 years $[2,6]$.

Subsequent analysis of data from Surveillance, Epidemiology, and End Results (SEER) Programme revealed that only $26.7 \%$ of US patients with lung cancer would have been eligible for screening by NLST criteria [7]. The use of multivariate tools that predict risk of lung cancer has been proposed as an alternative way to identify a high risk population for screening. The Liverpool Lung Project model (LLP) was developed from lung cancer cases and age and sex-matched population-based controls [8]. The UK Lung Screening (UKLS) Pilot screened participants whose 5-year lung cancer risk was greater than or equal to $5 \%$ as calculated by the LLP (version 2) model [9]. The PLCO $_{\mathrm{M} 2012}$ risk prediction model was derived and separately validated in participants in the Prostate, Lung, Colorectal and Ovarian (PLCO) cancer screening study [10]. A lung cancer risk of $\geqslant 1.51 \%$ over 6 years was identified as the threshold defining consistently reduced mortality with LDCT screening, and showed improved screening efficiency in one retrospective study [11].

We assessed the proportion of lung cancer patients diagnosed at four UK centres who would have fulfilled the various proposed criteria for screening at a time point 2 years before presentation. A 2-year interval was selected assuming a 4-year sojourn time for lung cancer [12], and represents a time at which the cancer might have been detectable by LDCT screening and earlier intervention may have improved outcomes.

This was a multicentre, prospective study. Data was routinely collected and used to calculate lung cancer risk in patients with suspected lung cancer following review in designated "fast-track" cancer clinics at four hospitals in West Yorkshire, UK (Bradford Teaching Hospitals NHS Foundation Trust, Calderdale and Huddersfield NHS Foundation Trust, Leeds Teaching Hospitals NHS Trust, and Mid Yorkshire Hospitals

@ERSpublications

The proportion of lung cancer patients eligible for screening 2 years before diagnosis varied between risk prediction tools. The highest proportion was identified with the $\mathrm{PLCO}_{\mathrm{M} 2012}$ score, detecting $\mathbf{4 4 . 1 \%}$ if screening up to 75 years or $58.6 \%$ to 80 years. http://bit.ly/2Jl2Got

Cite this article as: Gracie K, Kennedy MPT, Esterbrook G, et al. The proportion of lung cancer patients attending UK lung cancer clinics who would have been eligible for low-dose CT screening. Eur Respir J 2019; 54: 1802221 [https://doi.org/10.1183/13993003.02221-2018]. 
NHS Trust) from January to December 2016. Lung cancer diagnoses were either confirmed pathologically or made on the basis of clinical and radiological information following review at a lung cancer multidisciplinary meeting. Data was anonymised following confirmation of diagnosis, prior to analysis. Only data from patients subsequently diagnosed with lung cancer were included in the analysis. Risk data and criteria eligibility were calculated for a time point 2 years prior to the date of diagnosis (adjusted for age and smoking history). Thus, although the collection of data was prospective at the time of clinic attendance, the calculation of risk at the earlier time point was retrospective. Whether or not the patient would have fulfilled the NLST criteria and the USPSTF criteria (NLST extended to 80 years) was recorded. Lung cancer risk was calculated using the LLPv2 score [8] (risk over 5 years) and the PLCO M2012 $_{\text {score }}$ [10] (risk over 6 years). The formulae for the multivariate risk prediction tools have not been published, and these were calculated using the LLP MyLungRisk V2.0 website [13] and Brock University website [14], respectively.

All statistical analyses were carried out using the R statistical software package version 3.1.

Data were collected on 466 patients, of whom 347 were subsequently diagnosed with lung cancer. 16 patients were excluded due to incomplete data collection, providing a final study population of 331 patients. Demographic and clinical data are shown in table 1.

Considering screening eligibility 2 years prior to diagnosis, 115 (34.7\%, 95\% CI 29.8-40.0\%) patients would have fulfilled the NLST criteria, and 152 (45.9\%, 95\% CI 40.6-51.3\%) the USPSTF criteria. Of those excluded by age criteria, 36 patients (10.9\%) would have been under 55 years, and $41(12.4 \%)$ would have been over 80 years. The patients who would have been aged 55-80 years included 17 (6.7\%) never-smokers who are unlikely to be included in any screening programme according to currently proposed criteria.

Figure 1 shows the proportion of patients that would have been eligible at various risk thresholds using both LLPv2 and PLCO M2012 $_{2}$ models in two populations: ever-smokers aged 55-75 years, and ever-smokers aged 55-80 years. Using a LLPv2 5\% risk threshold (as in the UKLS pilot), 34.1\% (95\% CI 29.2-39.4\%) of all lung cancer patients would have been eligible for screening in the 55-75-year-old group, and 49.5\% (95\% CI 44.2-54.9\%) in the 55-80-year-old group. The Lung Screen Uptake Trial [15] used a lower LLP threshold of 2.5\%; the proportions eligible at this threshold were $41.4 \%$ (95\% CI $36.2-46.8 \%$ ) and $57.4 \%$ (95\% CI 52.0-62.6\%), respectively. Considering a 1.51\% threshold using the PLCO $_{\mathrm{M} 2012}$ score (used in the recent Manchester screening pilot [16]), the proportions eligible would have been $44.1 \%$ (95\% CI 38.9 49.5\%) and 58.6\% (95\% CI 53.2-63.8\%), respectively.

This is the first study to prospectively collect data to compare the performance of these two lung cancer risk prediction models, and to (retrospectively) assess what proportions of lung cancer patients would have been eligible for screening at a time point 2 years prior to diagnosis.

The proportion of patients who would have been eligible according to the NLST criteria is higher than the proportion reported from the SEER analysis (34.7\% versus $26.7 \%$ ) [7]. In the current study, the proportion eligible at an LLPv2 threshold of 5\% was similar to that seen with the NLST criteria with both proportions increasing when the upper age for screening is extended to 80 years. As expected, the proportion of patients eligible for screening increases if the LLPv2 risk threshold is dropped to $2.5 \%$. Of all proposed

TABLE 1 Population demographic and clinical data

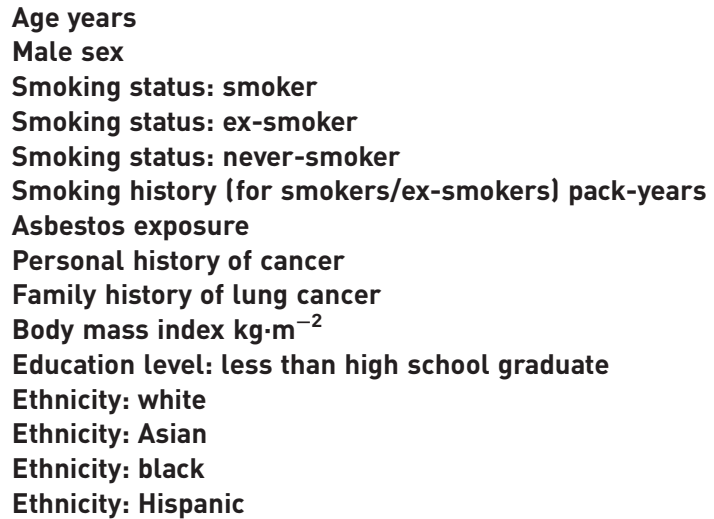

$69(62-77)$

$177(53.5 \%)$

$172(52.0 \%)$

$138(41.7 \%)$

$21(6.3 \%)$

$36(23-50)$

$77(23.3 \%)$

$56(16.9 \%)$

$58(17.5 \%)$

$25(22.1-28.4)$

$283(85.5 \%)$

$322(97.3 \%)$

$5(1.5 \%)$

$3(0.9 \%)$

$1(0.3 \%)$

Data are presented as medians with interquartile ranges or $\mathrm{n}(\%)$. 


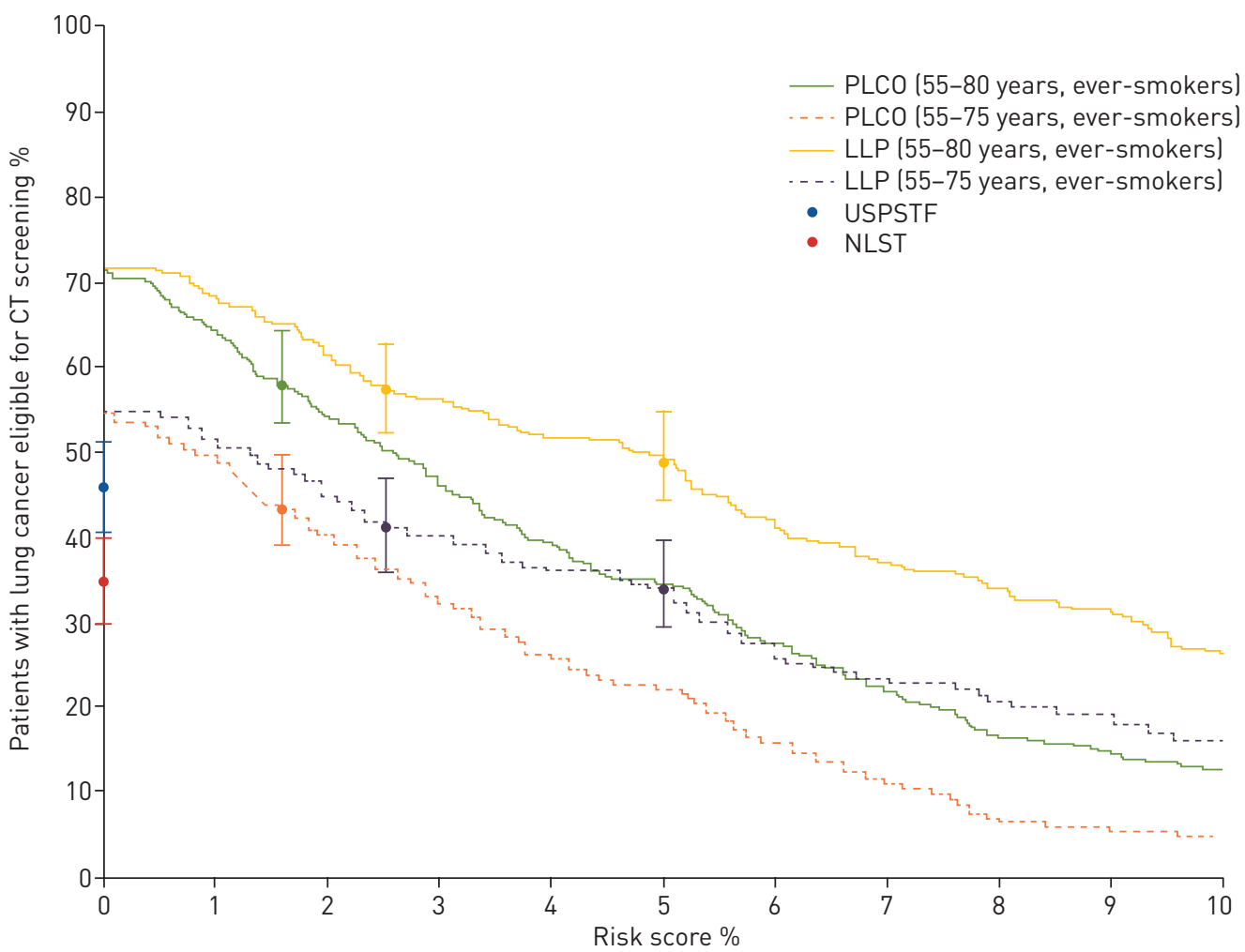

FIGURE 1 Eligibility for screening at different risk thresholds at the time-point 2 years prior to lung cancer diagnosis. The $\mathrm{x}$-axis shows Lung Cancer Risk Score as calculated by either Liverpool Lung Project (LLP) model version 2 (risk of developing lung cancer over the following 5 years) or the $\mathrm{PLCO}_{\mathrm{M} 2012}$ risk prediction model (risk of developing lung cancer over the following 6 years). The $y$-axis shows the proportion of lung cancer patients who would have been eligible for computed tomography (CT) screening 2 years earlier at different risk thresholds, and thus the lines on the graph show how the proportion eligible decreases as the risk score thresholds increase. The solid lines represent the proportions eligible when these risk scores are used in a population of 55-80-year-old ever-smokers; the dotted lines represent the proportions eligible in a population of 55-75-year-old ever-smokers. The $y$-axis intercept is less than $100 \%$ due to a proportion of lung cancer patients who would have been ineligible at any risk threshold by virtue of age leither too old or too young for consideration in a screening programmel or never-smoking status. The proportions of patients eligible by virtue of National Lung Screening Trial (NLST) criteria $1 \geqslant 30$ pack-years, $\leqslant 15$ years quit time, aged 55-74 years) and US Preventive Services Taskforce (USPSTF) criteria (as for NLST but extended to 80 years) are indicated by red and blue dots, respectively, on the y-axis. $95 \%$ confidence intervals are shown for NLST, USPSTF, LLPv2 at $2.5 \%$ and $5 \%$ thresholds, and $\mathrm{PLCO}_{\mathrm{M} 2012}$ at $1.51 \%$ threshold.

criteria, the highest proportion of lung cancer patients was identified using a $1.51 \%$ threshold with the $\mathrm{PLCO}_{\mathrm{M} 2012}$ score.

There are several weaknesses to this study. First, the number of patients analysed is relatively small (albeit from four centres); the performances of these criteria need comparing in larger populations across a greater number of sites to assess generalisability. Second, the study only considers patients presenting through lung cancer clinics. Many lung cancer patients present through other routes (e.g. emergency presentation) and the risk profile in these groups may differ and will not be reflected in the current analysis. Finally, this study only assessed the sensitivity of the proposed criteria for identifying patients with lung cancer. Of equal importance in determining the optimal threshold for screening is the proportion of people in the general population without lung cancer who would fulfil these criteria and receive a negative screening test, because an increase in screening sensitivity would invariably result in a decrease in specificity. Ongoing screening studies prospectively comparing these criteria in larger populations will yield relevant data in due course.

There is ongoing debate about the balance of benefits and harms, the cost-effectiveness of lung cancer screening in community practice, and the way to best deliver smoking cessation interventions alongside screening. These issues are not discussed here as this is outside the remit of the current analysis. Also, the presented analyses do not make any allowance for participation rates in screening programmes. Such rates need to be considered alongside the proportion of adults eligible for screening to estimate the number of 
early lung cancers that might be detected, the number of lung cancer deaths averted, and the number of overdiagnosed cases of cancer, were national programmes to be established in Europe.

In conclusion, risk models may show better sensitivity in selecting high risk patients for lung cancer screening than the criteria used in NLST. However, when used in a 55-75-year-old ever-smoking population, all proposed criteria identified fewer than half of the lung cancer patients in our analysis. Even if LDCT screening is implemented using these criteria, strategies are needed to ensure early detection in the majority of lung cancer patients who may not qualify for screening. Impact in this population requires earlier recognition and response to lung cancer symptoms. Lung cancer symptom awareness campaigns and strategies to reduce barriers to investigation of symptomatic patients have been associated with a reduction in advanced stage disease, although there is no definitive evidence of causation or impact on mortality [17].

Kathryn Gracie ${ }^{1}$, Martyn P.T. Kennedy ${ }^{2}$, Georgina Esterbrook ${ }^{3}$, Graham Smith ${ }^{3}$, Parry Blaxill ${ }^{3}$, Ali T. Ameri ${ }^{3}$, Kirsty I.A. Rodger ${ }^{2}$, Jonathan M. Robson ${ }^{2}$, Elankumaran Paramasivam ${ }^{2}$, Rehan Naseer ${ }^{4}$, Peter Smith ${ }^{5}$, Deborah A.B. Ellames ${ }^{2}$, Bana Hawramy ${ }^{2}$ and Matthew E.J. Callister ${ }^{2}$

${ }^{1}$ York Teaching Hospital NHS Foundation Trust, York, UK. ${ }^{2}$ Leeds Teaching Hospitals NHS Trust, Leeds, UK. ${ }^{3}$ Mid Yorkshire Hospitals NHS Trust, Wakefield, UK. ${ }^{4}$ Calderdale and Huddersfield NHS Foundation Trust, Huddersfield, UK. ${ }^{5}$ Airedale NHS Foundation Trust, Keighley, UK.

Correspondence: Martyn P.T. Kennedy, Leeds Teaching Hospitals NHS Trust, Respiratory Medicine, Level 4, Gledhow Wing, St James University Hospital, Beckett Street, Leeds, LS9 7TF, UK. E-mail: martyn.kennedy@nhs.net

Received: Nov 212018 | Accepted after revision: April 142019

Conflict of interest: None declared.

\section{References}

1 National Lung Screening Trial Research Team. Reduced lung-cancer mortality with low-dose computed tomographic screening. N Engl J Med 2011; 365: 395-409.

2 Moyer VA. U.S. Preventive Services Task Force. Screening for lung Cancer: U.S. Preventive Services Task Force Recommendation Statement. Ann Intern Med 2014; 160: 330-338.

3 Van Klaveren RJ, Oudkerk M, Prokop M, et al. Management of lung nodules detected by volume CT scanning. N Engl J Med 2009; 361: 2221-2229

4 De Koning H, Van Der Aalst C, Ten Haaf K, et al. Effects of volume CT lung cancer screening: mortality results of the NELSON randomised-controlled population based trial. (IASLC $19^{\text {th }}$ World Conference on Lung Cancer, Toronto, Canada, PL02.05) J Thorac Oncol 2018; 13: Suppl. 10, S185.

5 Oudkerk M, Devaraj A, Vliegenthart R, et al. European position statement on lung cancer screening. Lancet Oncol 2017; 18: e754-e766.

6 de Koning HJ, Meza R, Plevritis SK, et al. Benefits and harms of computed tomography lung cancer screening strategies: a comparative modeling study for the U.S. Preventive Services Task Force. Ann Intern Med 2014; 160: $311-320$

7 Pinsky PF, Berg CD. Applying the National Lung Screening Trial eligibility criteria to the US population: what percent of the population and of incident lung cancers would be covered? J Med Screen 2012; 19: 154-156.

8 Cassidy A, Myles JP, Van Tongeren M, et al. The LLP risk model: an individual risk prediction model for lung cancer. Br J Cancer 2008; 98: 270-276.

9 Field JK, Duffy SW, Baldwin DR, et al. The UK Lung Cancer Screening Trial: a pilot randomised controlled trial of low-dose computed tomography screening for the early detection of lung cancer. Health Technol Assess 2016; 20: $1-146$.

10 Tammemagi MC, Katki HA, Hocking WG, et al. Selection criteria for lung-cancer screening. N Engl J Med 2013; 368: 728-736.

11 Tammemagi MC, Church TR, Hocking WG, et al. Evaluation of lung cancer risks at which to screen ever- and never-smokers: screening rules applied to the PLCO and NLST cohorts. PLoS Med 2014; 11: e1001764.

12 Ten Haff K, Van Rosmalen J, De Koning HJ. Lung cancer detectability by test, histology, stage, and gender: estimates from the NLST and PLCO Trials. Cancer Epidemiol Biomarkers Prev 2015; 24: 154-161

13 Roy Castle Lung Cancer Foundation, Liverpool Lung Project (LLP). MyLungRisk V2.0. www.mylungrisk.org Date last accessed: March 4, 2019.

14 Brock University, Canada. Lung Cancer Screening and Risk Prediction: Lung Cancer Risk Calculators. https:// brocku.ca/lung-cancer-screening-and-risk-prediction/risk-calculators/ Date last accessed: March 4, 2019.

15 Quaife SL, Ruparel M, Beeken RJ, et al. The Lung Screen Uptake Trial (LSUT): protocol for a randomised controlled demonstration lung cancer screening pilot testing a targeted invitation strategy for high risk and "hard-to-reach" patients. BMC Cancer 2016; 16: 281.

16 Crosbie $\mathrm{PA}$, Balata $\mathrm{H}$, Evison $\mathrm{M}$, et al. Implementing lung cancer screening: baseline results from a community-based "Lung Health Check" pilot in deprived areas of Manchester. Thorax 2019; 74: 405-409.

17 Kennedy MPT, Cheyne L, Darby M, et al. Lung cancer stage-shift following symptom awareness campaign. Thorax 2018; 73: 1128-1136. 